\title{
Bipolarons in anisotropic crystals and low dimensional structures
}

\author{
N. I. Kashirina, E.V. Mozdor, E. A. Pashitskij, V. I. Sheka \\ Institute for Semiconductor Physics of NASU, 45, prospect Nauki, 252028 Kyiv, Ukraine
}

\begin{abstract}
We consider a simple bipolaron approach to description of anisotropic crystals in the strongcoupling limit. We have taken into account anisotropy of effective band masses and dielectric constants of crystals. The calculations of energy for two - electron system was fulfiled using wave functions chosen as a linear combination of gaussian orbitals, what enables us to easily estimate the electron correlation effects which can play a key role. Bipolaron formation should be quite possible in crystals with hightemperature superconductivity. We have considered an example of $\mathrm{La}_{2} \mathrm{CuO}_{4}$, in which there is a great anisotropy of effective masses and dielectric permittivities, where in the limiting case of the maximum anisotropy of effective masses $m_{x y}<<m_{z}$ the bipolaron binding energy is $25 \%$ of the polaron energy.
\end{abstract}

Keywords: bipolarons, anisotropic crystals, superconductivity.

Paper received 04.06.99; revised manuscript received 24.06.99; accepted for publication 12.07.99.

The bipolaron theory was first studied in [1] by the adiabatic approximation for the case of strong coupling of electronphonon interaction and isotropic continuum medium. Leaving aside the history of the bipolaron study presented in [2, 3], we consider the problem using the traditional Frohlich Hamiltonian which is generalized to the case of anisotropic two- atomic crystals $[4,5]$. For the bipolaron problem the corresponding Hamiltonian has the form

$$
\begin{aligned}
& \hat{H}_{12}=\hat{T}_{1}+\hat{T}_{2}+\hat{V}_{12}+\sum_{j} \int \hbar \omega_{j}(\mathbf{k}) a_{j}^{+}(\mathbf{k}) a_{j}(\mathbf{k}) d \mathbf{k}+ \\
& +4 \pi e^{2} \sum_{j, \alpha} \int \frac{d \mathbf{k}}{\mathbf{k} \hat{\varepsilon}_{e f}(\vec{k}) \mathbf{k}} \sqrt{\hbar / 2 \omega_{j}(\mathbf{k})} k_{\alpha} b_{\alpha} \times \\
& \times\left[\begin{array}{l}
a_{j}(\mathbf{k}) e_{j}^{\alpha}\left(\exp \left(i \mathbf{k} \mathbf{r}_{1}\right)+\exp \left(i \mathbf{k} \mathbf{r}_{2}\right)\right) \\
+a_{j}^{+}(\mathbf{k}) e_{j}^{* \alpha}\left(\exp \left(-i \mathbf{k} \mathbf{r}_{1}\right)+\exp \left(-i \mathbf{k} \mathbf{r}_{2}\right)\right)
\end{array}\right], \\
& \hat{T}_{i}=-\sum \frac{\hbar^{2}}{2 \mu_{\alpha}} \nabla_{i, \alpha}^{2}(i=1,2 ; \alpha=x, y, z), \\
& \hat{V}_{12}=\frac{e^{2}}{2 \pi^{2}} \int \frac{1}{\mathbf{k} \hat{\varepsilon}_{\infty} \mathbf{k}} \exp \left[i \mathbf{k}\left(\mathbf{r}_{1}-\mathbf{r}_{2}\right)\right] d \mathbf{k} .
\end{aligned}
$$

where $\hat{T}_{i}$ is the kinetic energy of the electron with coordinates $\mathbf{r}_{1}$ and $\mathbf{r}_{2}, \hat{V}_{1,2}$ is the potential energy of electrons, $\hat{\varepsilon}_{e f}^{-1}=\hat{\varepsilon}_{\infty}^{-1}-\hat{\varepsilon}_{0}^{-1}$.
The phonon frequencies $\omega_{j}(\mathbf{k})$ with normal coordinates $a_{\mathbf{k}}$ and $a_{\mathbf{k}}^{+}$, polarization vectors $\mathbf{e}_{j}(\mathbf{k})$ satisfy the dispersion equation

$$
\left(\omega_{j}^{2}(\mathbf{k})-\Omega_{\alpha}^{2}\right) e_{j}^{\alpha}=\frac{4 \pi}{\mathbf{k} \hat{\varepsilon}_{\infty} \mathbf{k}} k_{\alpha} b_{\alpha} \sum\left(k_{\beta} b_{\beta}\right) e_{j}^{\beta},
$$

where

$b_{\alpha}=\frac{1}{4 \pi}\left(\varepsilon_{0, \alpha}-\varepsilon_{\infty, \alpha}\right)^{1 / 2} \Omega_{\alpha}$

Polarization vectors follow the condition

$\sum e_{j}^{\alpha}(\mathbf{k}) e_{j}^{* \beta}(\mathbf{k})=\delta_{\alpha, \beta} ; \sum e_{j}^{\alpha}(\mathbf{k}) e_{i^{\mathrm{B}}}^{* \alpha}(\mathbf{k})=\delta_{j, i}$.

It should be noted that the Hamiltonian in Eq.(1) describes the bipolaron states in crystals with the uniaxial anisotropy. In this case, if we reduce the tensors of effective mass and dielectric permittivity $\hat{\varepsilon}_{0}$ and $\hat{\varepsilon}_{\infty}$ to the main axes, notations $m_{x x}=m_{y y}=m_{\perp}, m_{z z}=m_{\|}$can be introduced.

Let us assume $\varepsilon_{x x}=\varepsilon_{y y}=\varepsilon_{\perp}, \quad \varepsilon_{z z}=\varepsilon_{\|}$.In contrast to works $[6,7]$ where the limiting cases of low- dimensional systems are considered, we study crystals with arbitrary anisotropy of effective band mass and dielectric permittivity (in the general case we also take into account their time and space dixspersion ).

In the weak -coupling limit there are no localized bipolaron states in the continuum medium for which the 


\section{N. I. Kashirina et al.: Bipolarons in anisotropic crystals and low ...}

Hamiltonian used Eq.1 is valid.

Let us use unitary transformation to change $a_{j}(\mathbf{k}) \rightarrow a_{j}(\mathbf{k})+f_{j \mathbf{k}}$, being $f_{j \mathbf{k}}$ independent of variables $\mathbf{r}$ and $a_{j}(\mathbf{k})$. The average on the phonon vacuum leads to the Hamiltonian

$$
\begin{aligned}
& \bar{E}=\bar{T}_{1}+\bar{T}_{2}+\bar{V}_{12}+\sum \int \hbar \omega_{j} f_{j k} f_{j k}^{*} d \mathbf{k}+ \\
& +\mathbf{4} \pi \mathbf{e}^{2} \sum \int \frac{d \mathbf{k}}{\mathbf{k} \hat{\varepsilon}_{e f} \mathbf{k}} \sqrt{\hbar / 2 \omega_{j}(\mathbf{k})} \times \\
& \times\left(\left\langle e^{i \mathbf{k} \mathbf{r}_{1}}\right\rangle+\left\langle e^{i \mathbf{k} \mathbf{r}_{2}}\right\rangle\right) \cdot\left(f_{j k} e_{j}^{\alpha}(\mathbf{k})+f_{j k}^{*} e_{j}^{* \alpha}(\mathbf{k})\right) .
\end{aligned}
$$

Minimization of Eq.(6) over $f_{j k}$ and summation over the polarization vectors (which is similar to minimization of the polaron energy, developed in [5]) result in the adiabatic Pekar bipolaron functional

$$
J(\Psi)=\bar{T}_{1}+\bar{T}_{2}+\bar{V}_{12}+\bar{V}_{e f},
$$

where

$$
\begin{aligned}
& \bar{V}_{e f}=V_{e f}\left(\hat{\varepsilon}_{\infty}\right)-\bar{V}_{e f}\left(\hat{\varepsilon}_{0}\right), \quad \bar{V}_{12}=\int \frac{P(\mathbf{k},-\mathbf{k}) d \mathbf{k}}{\mathbf{k} \hat{\varepsilon}_{\infty} \mathbf{k}} ; \\
& \bar{V}_{e f}(\hat{\varepsilon})=\int \frac{P(\mathbf{k}, 0)-\left.P(0, \mathbf{k})\right|^{2} d \mathbf{k}}{\mathbf{k} \hat{\varepsilon} \mathbf{k}}, \\
& P\left(\mathbf{k}_{1}, \mathbf{k}_{2}\right)=\left\langle\Psi\left(\mathbf{r}_{1}, \mathbf{r}_{2}\right)\left|\exp \left(i \mathbf{k}_{1} \mathbf{r}_{\mathbf{1}}+i \mathbf{k}_{2} \mathbf{r}_{2}\right)\right| \Psi\left(\mathbf{r}_{1}, \mathbf{r}_{2}\right)\right\rangle .
\end{aligned}
$$

Good results were obtained in [8] by minimization of functional in Eq.(7) for isotropic systems, where the trial wave functions were taken in the form

$$
\Psi\left(\mathbf{r}_{1}, \mathbf{r}_{2}\right)=A\left(1+\beta r_{12}\right) \exp \left(-\alpha \mathbf{r}_{1}-\alpha \mathbf{r}_{2}\right) .
$$

Here $r_{12}=\left|\mathbf{r}_{\mathbf{1}}-\mathbf{r}_{2}\right|$, and $\mathbf{r}_{\mathbf{1}}, \mathbf{r}_{2}$ are the coordinates of the first and the second electron, respectively. The account of the term with multiplier $\beta r_{12}$ enables us to consider the electron correlation effects. But in the case of anisotropic crystals, the use of these wave functions leads to cumbersome calculations when evaluating integrals for potential energy $\bar{V}_{12}$ and the term corresponding to the electron-phonon interaction. However, using a set of Gaussian trial wave functions, we made the analytical calculations in the explicit form.

In this paper, we use the trial wave function, which is axially symmetric and has the form

$$
\begin{aligned}
& \Psi\left(\mathbf{r}_{1}, \mathbf{r}_{2}\right)=\sum C_{j} \exp \left(-a_{1 j} z_{1}^{2}-2 a_{2 j} z_{1} z_{2}-a_{3 j} z_{2}^{2}\right) \times \\
& \times\left(\exp \left(-b_{1 j} \rho_{1}^{2}-2 b_{2 j} \rho_{1} \rho_{2}-b_{3 j} \rho_{2}^{2}\right) .\right. \\
& \text { where } \boldsymbol{\rho}=\{x, y\} .
\end{aligned}
$$

In this relation, the parameters are chosen to account the symmetry or antisymmetry with respect to exchange of electron coordinates.

The interference terms $2 a_{2 i} z_{1} z_{2}$ and $2 b_{2 i} \bar{\rho}_{1} \bar{\rho}_{2}$ in the exponential index take into account the correlation effects.

To calculate the minimum of functional Eq. (7), it is convenient to make the scale transformation $\mathbf{r} \Rightarrow \lambda \mathbf{r}$, where $\lambda$ is the scale multiplier, which can be found by the virial theorem, $\lambda=-\left(\bar{V}_{12}+\bar{V}_{e f}\right) / 2 \bar{T}$, and $E_{\lambda}=-\left(\bar{V}_{12}+\bar{V}_{e f}\right) / 4 \bar{T} N$, where $N$ is the normalization multiplier.

All averaged quantities in the functional Eq.7 can be easily calculated by the wave functions Eq. 9. The minimization of $\bar{E}_{\lambda}$ with respect to the parameters $C_{j}, a_{i j}, b_{i j}(i=1,2,3 ; j=1, \ldots, n)$ was made by the method of fast descent and it was applied to calculation of the energy of para- and orto- helium ground states. The calculations for $n=7$ yield the following results $E_{0}=$ -2.8722374 for the parahelium, and $E_{1}=-2.1183365$ for the ortohelium, which coincide with the best calculations of corresponding quantities [9] in all significant numerals. This application shows that our approximation of trial functions by Eq.(9) is rather convenient to minimize the bipolaron functional.

We note that the choice of the wave functions in the form of Eq. (9) enables us to perform easily the symmetrization procedure (or antisymmetrization) over the electrons coordinates. For instance, for the triplet states the coefficients $C_{j}$ must differ in sign for terms different in the exchanging electron coordinates $\mathbf{r}_{1}$ and $\mathbf{r}_{2}$, while for the singlet states they coincide in sign. The following symmetrization (the choice equivalent variational parameters corresponding to the terms, which are different in exchanging electron coordinates) can be made by the minimization of the functional. The possibility of calculation of singlet- triplet splitting for twoelectron system by wave functions chosen as linear combination of gaussian orbitales enables us to estimate the dependence of the exchange energy on the inter -ion distance without ignoring the electron correlation effects which can play a key role.

Fig 1. shows the dependence of the bipolaron ground state energy (line 1) and the polaron one (line 2) on the ratio of effective masses (in logarithmic scale) for the cases of anisotropy «light axis» $\left(m_{\perp} / m_{\|}>1\right)$ and light plane $\left(m_{\perp} / m_{\|}<1\right)$, when $\varepsilon_{\infty} / \varepsilon_{0} \Rightarrow 0$.

The corresponding bipolaron binding energy is plotted in Fig. 2. The bipolaron and polaron energies of ground state are shown to decrease if the anisotropy rises, while the polaron binding energy increases (especially for crystals with quassi-one-dimensional anisotropy).

In the isotropic case, we result in lower energy of the bipolaron ground state and the larger binding energy $\Delta W$ $\left(\Delta W / 2 I_{p} \cong 0.253\right.$ as $\left.\varepsilon_{\infty} / \varepsilon_{0} \Rightarrow 0\right)$ then the best results [8] calculated for the strong-coupling case, with the use of the conventional trial wave functions Eq. 8. The minimization of the polaron functional by trial function involving seven gaussian orbitales exactly leads to the energy evaluated in [10] in strong - coupling limit. We use this value to evaluate the binding energy. The situation is similar to the case of isotropic crystals.

In comparison with isotropic case, the regions of parameter $\eta=\varepsilon_{\infty} / \varepsilon_{0}$, where bipolaron exists, are slightly extended. For example, if $m_{\perp} / m_{\|}=32$ the bipolaron is stable as 


\section{N. I. Kashirina et al.: Bipolarons in anisotropic crystals and low ...}

$0 \leq \eta \leq \eta_{c}=0.150$, and if $m_{\perp} / m_{\|}=64$ it yields $\eta_{c}=0.140$.

We note that the calculation of the bipolaron energy in the ground state for anisotropic crystals is similar to the calculations for the crystals with anisotropic effective masses, which we study. Moreover, in the limit $\varepsilon_{\infty} / \varepsilon_{0} \rightarrow 0$, using the scale transformation of electron coordinates $\left(z_{1,2} \rightarrow \sqrt{\varepsilon_{\perp} / \varepsilon_{\|}} \tilde{z}_{1,2}\right.$ we can reduce the problem to the case under consideration, where effective masses are anisotropic, while the dielectric constants are isotropic. The new effective masses are connected with the initial dielectric constants. by the relation $\tilde{m}_{\perp} / \tilde{m}_{\|}=\varepsilon_{\infty \|} / \varepsilon_{\infty \perp}$.

To discuss the bipolaron formation in the crystals where high-temperature conductivity is observed, we consider example $\mathrm{La}_{2} \mathrm{CuO}_{4}$, in which there is a great anisotropy of effective masses and dielectric permittivities. This crystal provides a good possibility for calculation of the bipolaron bound energy in the crystals where the anisotropy of effective masses and dielectric constants occur coincidentally. In our assumption, the data of static and high- frequency dielectric constants are only required, these constants are well-known from experiments [11], $\varepsilon_{\infty}=4, \varepsilon_{0}=50$ in the plane of layers $\mathrm{CuO}_{2}$, and $\varepsilon_{0}=23$ in the perpendicular direction.

In the case of isotropic effective masses, the bipolaron binding energy is $15.6 \%$ of the polaron energy, but in the limiting case of the maximum anisotropy of effective masses $m_{x y}<<m_{z}$, this quantity is $25,2 \%$ of the polaron energy. Therefore bipolaron formation should be quite possible in crystals with high-temperature conductivity, especially, if we take into account that these crystals are the systems with «light plane» anisotropy.

Fig. 3 shows the bipolaron bound energies expressed in the units of the double polaron energy at various values of the ratio $m_{x y} / m_{z}$. The lines $1,2,3,4,5$, correspond to this ratio at $1,2^{-2}, 2^{-4}, 2^{-15}, 2^{-20}$, respectively.

It is seen that the region of bipolaron existence extends,

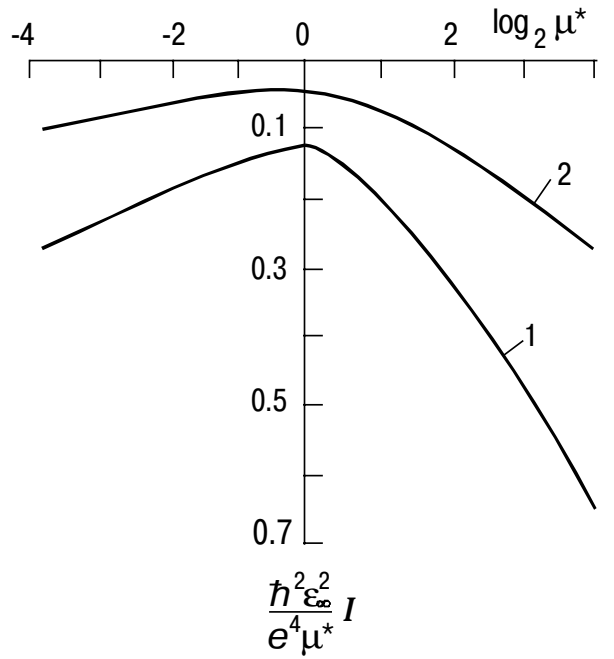

Fig. 1. Energy of polaron ground state (line 1), and bipolaron ground state (line 2). $m^{*}=\min \left\{m_{x y}, m_{z}\right\}$.

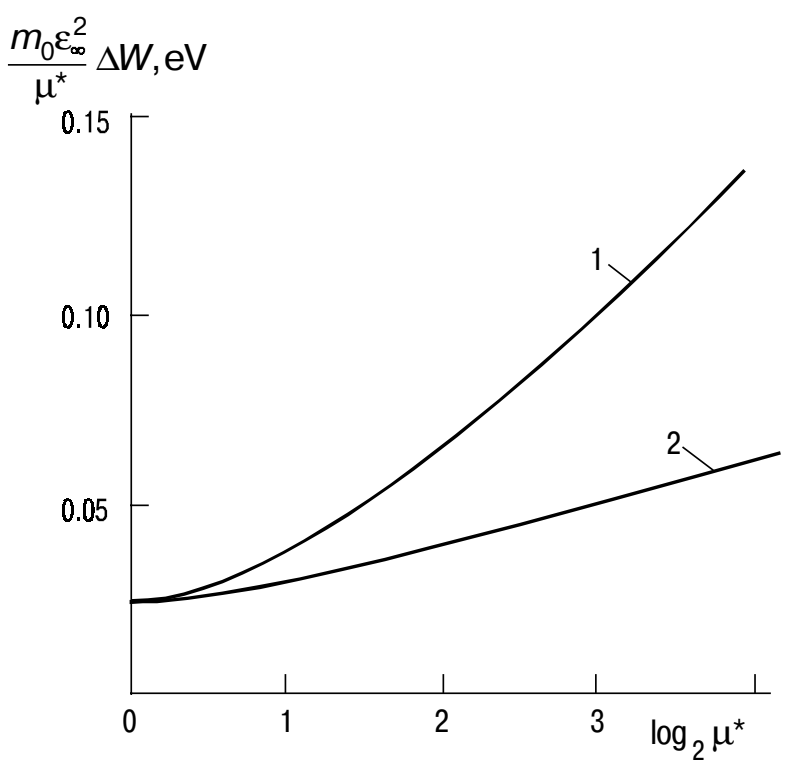

Fig. 2. Bipolaron binding energy for the anisotropic cases: «light axis» $\left(m^{*}=m_{z}\right)$, and «light plane» $\left(m^{*}=m_{x y}\right)$.

and the binding energy increases as the anisotropy of crystals grows. We point out that we can consider the two-dimensional systems by this method using scale transformation $I_{p}(2 D) / I_{p}(3 D) \cong 2 / 3(3 \pi / 4)^{2}$ (where $I_{p}(2 D)$ and $I_{p}(3 D)$ are the polaron energies in the two- and three- dimensional systems, respectively). Similar relations can be derived for the bipolaron ground state energy and, conse-

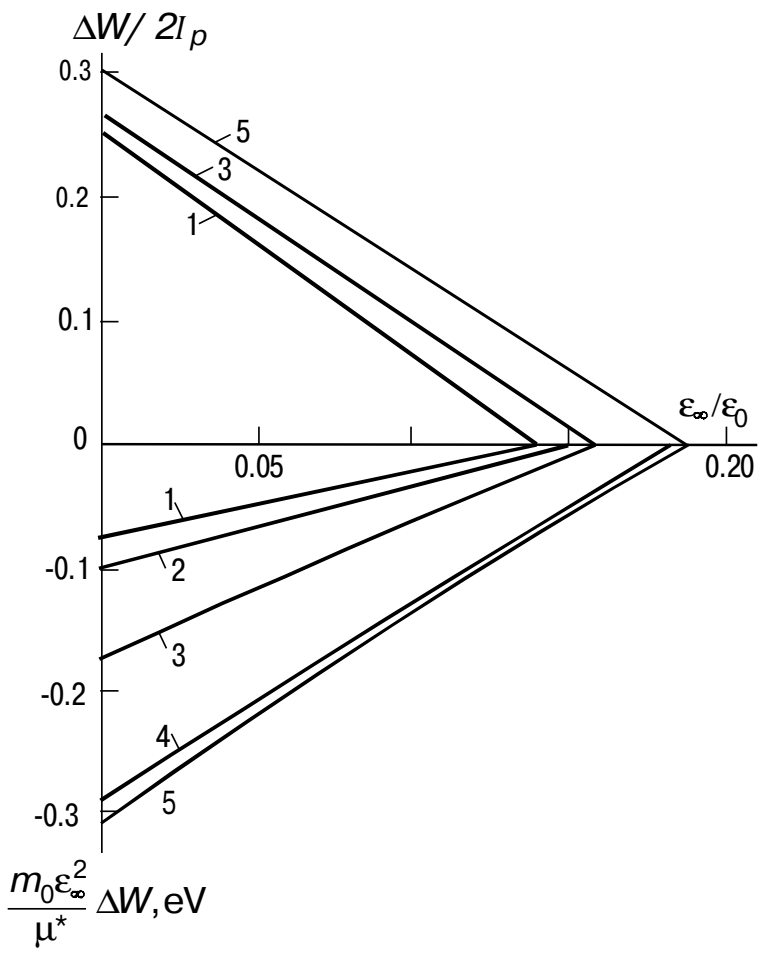

Fig. 3. Dependence of bipolaron binding energy on parameter $\eta_{c}=\varepsilon_{\infty} / \varepsilon_{0}$. The lines $1,2,3,4,5$, correspond to the parameter $m_{x y} / m_{z}$ equal to $1,2^{-2}, 2^{-4}, 2^{-15}, 2^{-20}$, respectively. 


\section{N. I. Kashirina et al.: Bipolarons in anisotropic crystals and low ...}

quently, for the considered bipolaron binding energy. The validity of this scale transformation in the case of two-dimensional systems is revealed in [12], and numerically tested by us for the case of the maximum anisotropy of effective masses.

It must be noted note that we consider electron- opticalphonon interaction. Using the continuum approximation, we can easily express the binding energy for electron- acoustic-phonon interactions. But the absence of reliable data on the tensor of the deformational potential makes it impossible to calculate the binding bipolaron energy with account of the condenson effect. We note that this effect can play a key role due to the increasing bound energy in this case. The binding energy of autolocalized state may change significantly as a result of interaction with plasma vibrations of charge carries in conduction and valence bands, or in systems of movable ions for crystals with ion conductivity [13]. But in addition to the effect caused by screening Coulomb interactions of electrons and ion vacancies, for bounded systems, such as polarons, bipolarons, $\mathrm{F}, \mathrm{F}^{\prime}, \mathrm{F}_{2}$ - centers there is a screening of electron interactions with optical phonons. There for, without detailed calculations of binding energy for the polaron and the bipolaron surrounded by plasmons we cannot estimate the plasmon effect on these energies.

\section{References}

1. S. I. Pekar, Studies in the Electronic Theory of Crystals. Gostekhizdat, Moscow (1951), 256 p., (in Russian).

2. G. Verbist, F. M. Peeters and J. T. Devreese, Possible (bi) polaron effects in the high $-\mathrm{T}_{\mathrm{C}}$ superconductors // Phys. Scripta. T39, pp. $66-70,(1991)$
3. V. I. Vinitskij, N. I. Kashirina, E. A. Pashitsky, Bipolaron states of great radius and the problem of high temperature superconductivity // Ukr. Fiz. Zh. 37(1), pp.76 -94 (1992).

4. E. Bergman, Phenomenological theory of the interaction of electrons with polar phonons in anisotropic crystals // J. Phys. and Chem. Sol. 35(10), pp. 1433-1436 (1974).

5. V. I. Orbukh and V. I. Sheka, Conntinualny polyaron sil'noy svyazy v anizotropnom krystalle // Ukr. Fiz. Zh. 30(5), pp.771775 (1985) (in Russian).

6. J. T. Devreese, Polaron physics in 2D and 3D // Phys. Scripta. T25, pp. 309 - 315 (1989).

7. E. P. Pokatilov, S. I. Beril, V. M. Fomin and G. Yu. Ryabukhin, Polaron pairing in multi- layer structures // Phys. Status. Sol. (b). 171(2), pp. 437 - 441 (1992).

8. S. G. Suprun and B. Ya. Moizhes, O roly elektronnoy korrelyatcii v obrazovanii bypolyaritona Pekara// Fiz. Tverd. Tela. 24(5), pp. 1571-1573 (1982) (in Russian).

9. P. Gombash, Many Particle Problem in Quantum Mechanics. Inost. Lit., Moscow (1953) (in Russian).

10. S. J. Miyake, strong-coupling limit of the polaron ground state // J. Phys. Soc. Jpn. 38(1), pp.181- 182 (1975).

11. D. Reagor, E. Ahrens, S-W. Cheong, A. Migliori and Z. Fisk, Large dielectric constants and massive carriers in $\mathrm{La}_{2} \mathrm{CuO}_{4} / /$ Phys. Rev. Lett. 62(17), pp.2048 -2051(1989).

12. G. Verbist, F. M. Peeters and J. T. Devreese, Large bipolarons in two and three dimensions // Phys.Rev. B 43 (4), pp. 2712- 2720 (1991)

13. V. D. Lakhno, Continuum excitons in semiconductors // Phys. Rev. B 46(12), pp. $7519-7527$ (1992). 